\title{
BMJ Open Bacteremia Antibiotic Length Actually Needed for Clinical Effectiveness (BALANCE) randomised clinical trial: study protocol
}

\author{
Nick Daneman (D) , ${ }^{1}$ Asgar H Rishu, ${ }^{2}$ Ruxandra L Pinto, ${ }^{3}$ Yaseen M Arabi, ${ }^{4}$ \\ Deborah J Cook, ${ }^{5}$ Richard Hall, ${ }^{6}$ Shay McGuinness, ${ }^{7}$ John Muscedere, ${ }^{8}$ \\ Rachael Parke, ${ }^{9}$ Steven Reynolds, ${ }^{10}$ Benjamin Rogers, ${ }^{11}$ Yahya Shehabi, ${ }^{12}$ \\ Robert A Fowler, ${ }^{13}$ On behalf of the Canadian Critical Care Trials Group
}

To cite: Daneman N, Rishu AH, Pinto RL, et al. Bacteremia Antibiotic Length Actually Needed for Clinical Effectiveness (BALANCE) randomised clinical trial: study protocol. BMJ Open 2020;10:e038300. doi:10.1136/ bmjopen-2020-038300

- Prepublication history and additional material for this paper are available online. To view these files, please visit the journal online (http://dx.doi. org/10.1136/bmjopen-2020038300).

Received 06 March 2020 Revised 30 March 2020 Accepted 14 April 2020

Check for updates

(C) Author(s) (or their employer(s)) 2020. Re-use permitted under CC BY-NC. No commercial re-use. See rights and permissions. Published by BMJ.

For numbered affiliations see end of article.

Correspondence to

Dr Nick Daneman;

Nick.Daneman@sunnybrook.ca

\section{ABSTRACT}

Introduction Bloodstream infections are a leading cause of mortality and morbidity; the duration of treatment for these infections is understudied.

Methods and analysis We will conduct an international, multicentre randomised clinical trial of shorter (7 days) versus longer (14 days) antibiotic treatment among hospitalised patients with bloodstream infections. The trial will include 3626 patients across 60 hospitals and 6 countries. We will include patients with blood cultures confirming a pathogenic bacterium after hospital admission. Exclusion criteria will include patient factors (severe immunosuppression), infection site factors (endocarditis, osteomyelitis, undrained abscesses, infected prosthetic material) and pathogen factors (Staphylococcus aureus, Staphylococcus lugdunensis, Candida and contaminant organisms). We will leave the selection of specific antibiotics, doses and route of delivery to the discretion of treating physicians; no placebo control will be used given the diversity of pathogens and sources of bacteraemia. The intervention will be assignment of treatment duration to be 7 versus 14 days. We will minimise selection bias via central randomisation with variable block sizes, with concealed allocation until day 7 of adequate antibiotic treatment. The primary outcome is 90-day survival; we will test whether 7 days is noninferior to 14 days of treatment, with a non-inferiority margin of $4 \%$ absolute mortality. Secondary outcomes include hospital and intensive care unit (ICU) mortality, relapse rates of bacteraemia, hospital and ICU length of stay, mechanical ventilation and vasopressor duration, antibiotic-free days, Clostridium difficile infection, antibiotic allergy and adverse events and colonisation/ infection with antibiotic-resistant organisms.

Ethics and dissemination The study has been approved by the ethics review board at each participating site. Sunnybrook Health Sciences Centre is the central ethics committee. We will disseminate study results via the Canadian Critical Care Trials Group and other collaborating networks to set the global paradigm for antibiotic treatment duration for non-staphylococcal Gram-positive, Gram-negative and anaerobic bacteraemia, among patients admitted to hospital.

\section{Strengths and limitations of this study}

- The Bacteremia Antibiotic Length Actually Needed for Clinical Effectiveness (BALANCE) study is the largest randomised clinical trial ever conducted among patients with bloodstream infection and should set the paradigm for antibiotic treatment duration for these patients.

- BALANCE will provide generalisable results by including a wide array of bloodstream pathogens and underlying sources of infection, examining both critically and non-critically ill hospitalised patients, and including sites across six countries with varying baseline antibiotic resistance rates.

- If 7 days of antibiotic treatment is non-inferior to 14 days of treatment, this could lead to reductions in global antibiotic use, costs and antibiotic-related complications, including adverse events, Clostridium difficile and antibiotic resistance.

- The diversity of pathogens and underlying infections that cause bloodstream infection render placebo controls infeasible. We will minimise bias through central randomisation, allocation concealment until day 7 , an objective primary outcome and blinded adjudication of other outcomes such as relapse and secondary infections with antibiotic-resistant organisms.

Trial registration number The BALANCE (Bacteremia Antibiotic Length Actually Needed for Clinical Effectiveness) trial was registered at www.clinicaltrials.gov (registration number: NCT03005145).

\section{INTRODUCTION}

There are more than 600000 episodes of bloodstream infection per year in North America, and more than 1200000 episodes in Europe. ${ }^{1}$ These infections affect $15 \%$ of critically ill patients, result in prolongation of hospital stay, excess healthcare costs and a substantial mortality. ${ }^{2-5}$ 
Hospitals, and intensive care units (ICUs) in particular, are the location of greatest antimicrobial pressure; however, audits indicate that $30 \%-50 \%$ of antibiotic use in acute care and critical care settings are unnecessary or inappropriate ${ }^{6-8}$ and leads to avoidable costs and complications. Antibiotics are among the most common cause of serious adverse drug events, ${ }^{9}$ which occur in up to $5 \%-10 \%$ of inpatient recipients. ${ }^{10}$ Excessive durations of antibiotic therapy are the largest contributor to inappropriate antibiotic use in acute care hospitals, long-term care facilities and ambulatory clinics. ${ }^{71-13}$ Discontinuing antibiotics after achieving clinical cure can potentially reduce the burden of adverse events, Clostridium difficile infections and selection of antibiotic-resistant pathogens. $^{1415}$

Meta-analysis of randomised clinical trials (RCTs) has demonstrated that shorter duration antibiotic treatment is as effective as longer duration treatment for a range of mild to moderate infections. ${ }^{16}$ Even in critically ill patients with ventilator-associated pneumonia, mortality rates and relapse rates were non-inferior among the 402 patients randomised to receive shorter ( 8 day) versus longer ( 15 day) courses of antibiotics. ${ }^{17}$ However, similar high-grade evidence is lacking for the treatment of critically ill patients with bloodstream infections. ${ }^{2} 1819$ One recent study has examined 7 versus 14 days of treatment for bacteraemia in non-critically ill patients and has suggested that this may be a safe approach, but used a wide non-inferiority margin and was limited to infections with Gram-negative pathogens. ${ }^{20}{ }^{21}$ Specific guidelines for treatment durations exist for pneumonia, ${ }^{22}{ }^{23}$ intra-abdominal infection, ${ }^{24}$ catheter-related bloodstream infection, ${ }^{25}$ pyelonephritis ${ }^{26}$ and skin and soft tissue infection ${ }^{27}$ but no guidelines exist for the optimal duration of treatment for the subset of patients with bacteraemia.

We have performed a systematic review of the existing literature ${ }^{19}$ practice surveys of infectious diseases and critical care physicians, ${ }^{28}{ }^{29}$ a single-centre ${ }^{30}$ and multicentre observational study, ${ }^{31}$ which collectively identified gaps in current evidence, extensive practice variation and equipoise for a randomised trial comparing shorter (7 days) versus longer (14 days) antibiotic treatment durations for bloodstream infections. Through the Bacteremia Antibiotic Length Actually Needed For Clinical Effectiveness (BALANCE) pilot RCT (ClinicalTrials.gov identifier: NCT02261506), we documented the feasibility of this trial design among 115 patients in ICUs, thereby providing a vanguard for the BALANCE main trial. ${ }^{32} \mathrm{We}$ have subsequently confirmed the feasibility of enrolling patients on non-ICU wards in a parallel pilot RCT (ClinicalTrials.gov identifier NCT02917551), facilitating expansion of the BALANCE trial to include non-ICU patients. ${ }^{33}$

The primary aim of the BALANCE RCT will be to determine whether 7 days (as compared with 14 days) of adequate antibiotic treatment is associated with noninferior 90-day survival for hospitalised patients with bacteraemia.

\section{METHODS AND ANALYSIS}

\section{Study design}

We will conduct a multicentre randomised concealed allocation trial of shorter duration (7 days) versus longer duration (14 days) antibiotic treatment for patients with bacteraemia admitted to hospital.

\section{Setting}

The BALANCE trial (balance.ccctg.ca) is currently being conducted across a geographically and clinically diverse spectrum of ICUs and hospitals in Canada (currently 36 sites), Australia (6 sites), New Zealand (10 sites), the USA (2 sites), Saudi Arabia (2 sites) and Israel (1 site). We commenced enrolment at the central study site, Sunnybrook Health Sciences Centre in Toronto, Canada, at the beginning of the vanguard pilot (October 2014) and then added each additional site in a staggered fashion after ethics approval, contract and site initiations were accomplished. We continue to welcome new sites into BALANCE and anticipate approximately 60-70 active sites by the time of trial completion.

\section{Participants}

Hospitalised patients will be considered for enrolment in this study if they meet all inclusion and no exclusion criteria.

\section{Inclusion criteria}

Patient is admitted to hospital at the time a blood culture result is reported as positive with a pathogenic bacterium.

\section{Exclusion criteria}

1. Patient already enrolled in the trial.

2. Patient has severe immune system compromise, as defined by: absolute neutrophil count $<0.5 \times 10^{9} / \mathrm{L}$ or is receiving immunosuppressive treatment for solid organ or bone marrow or stem cell transplant.

3. Patient has a prosthetic heart valve or synthetic endovascular graft (post major vessel repair with synthetic material; coronary artery stents are not an exclusion).

4. Patient has a documented or strong suspicion of a syndrome with well-defined requirement for prolonged treatment: (1) infective endocarditis, (2) osteomyelitis/septic arthritis, (3) undrainable/undrained abscess and (4) unremovable/unremoved prostheticassociated infection (eg, infected pacemaker, prosthetic joint infection, ventriculoperitoneal shunt infection). Central venous catheters, including tunnelled central intravenous catheter and urinary catheters are not excluded.

5. Patient has a single positive blood culture with a common contaminant organism according to Clinical and Laboratory Standards Institute guidelines: coagulasenegative staphylococci, Bacillus spp, Corynebacterium spp, Propionobacterium spp, Aerococcus spp or Micrococcus spp. ${ }^{34}$

6. Patient has a positive blood culture with S. aureus $^{35}$ or S. lugdunensis. 


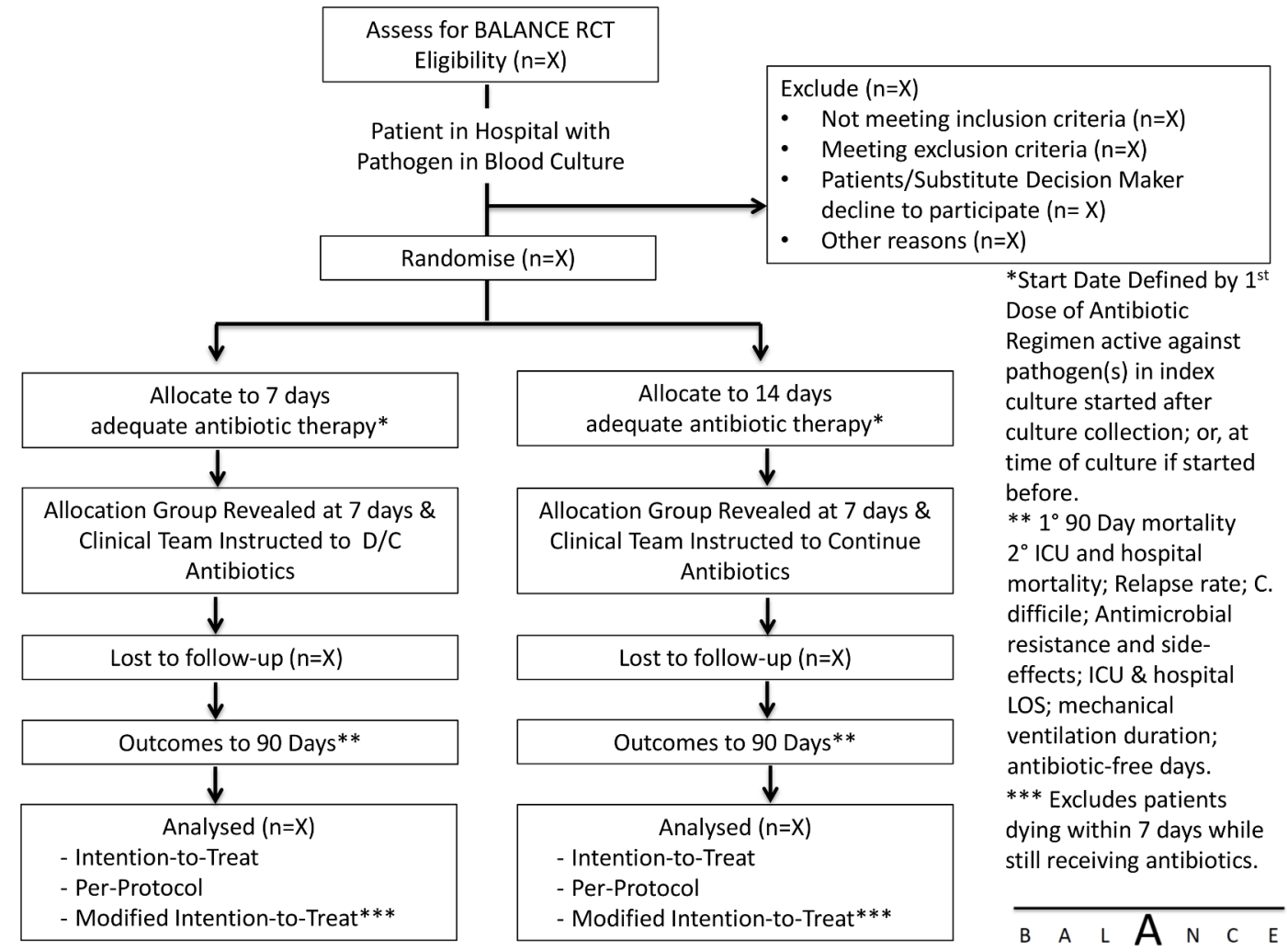

Figure 1 BALANCE RCT intervention flow diagram. BALANCE, Bacteremia Antibiotic Length Actually Needed for Clinical Effectiveness; C. difficile, Clostridium difficile; D/C, discontinue; ICU, intensive care unit; LOS, length of stay; RCT, randomised clinical trial.

7. Patient has a positive blood culture with rare bacterial pathogens requiring prolonged treatment (eg, Mycobacteria spp, Nocardia spp, Actinomyces spp, Brucella spp, Burkholderia pseudomallei).

8. Patient has a positive blood culture with Candida spp or other fungal species.

\section{Trial intervention}

We will randomise patients to receive a shorter duration of adequate antibiotic therapy ( 7 days) versus a longer duration (14 days) (figure 1). Adequate antibiotic treatment will be defined as treatment with an antibiotic (or antibiotics) to which the local laboratory has reported the $\operatorname{organism}(\mathrm{s})$ responsible for the bloodstream infection as susceptible. The duration of adequate treatment will be determined as the cumulative number of days on which at least one dose of adequate treatment is delivered beyond the date of collection of the index blood culture specimen. ${ }^{36}$ The selection of specific antimicrobial agent(s), doses and route of delivery will be at the discretion of the treating clinical team. The research team at each site will visit daily to ensure that antibiotics are stopped at the prespecified date (end of day 7 or 14).

\section{Randomisation and allocation concealment}

We will use web-based randomisation through RANDOMIZE.NET (http://www.randomize.net/), with variable block sizes, stratified by hospital site and by ICU versus non-ICU location. After the full susceptibility results become available, the site research coordinator along with site coinvestigators will determine the date for day 7 unblinding, taking into account the number of days that the patient has already received adequate antibiotics after the blood culture collection date. To avoid differentially influencing antibiotic choices and clinical decision-making, the randomisation assignment will not be communicated to any clinical staff or research personnel (research coordinator, study critical care or infectious diseases investigators) - until the end of day 7 . At day 7, another email will be sent with the unblinded treatment assignment for the patient to the site research coordinator. If a patient is randomised to the short (day) treatment arm, the treating team will be informed to stop the antibiotics at the completion of 7 days of antibiotics appropriate for the causative pathogen; if the patient is randomised to the long (day 14) arm, the team will be instructed to continue the antibiotic until that date, including beyond hospital discharge if necessary.

\section{Mechanistic substudies}

Biomarker substudy

Blood samples will be drawn on the randomisation day and at days 7, 10 and 14 from the index blood culture collection to measure procalcitonin (PCT) levels along with other novel inflammatory and angiogenic biomarkers (soluble triggering receptor expressed on myeloid cells (s-TREM-1), tumor necrosis factor receptor 
(S-TNFR-1 and S-TNFR-2), interleukin 6, Chitinase-3like protein (CHI3L-1), angiopoietin (Ang)-1, Ang-2, tyrosine kinase with immunoglobulin (sTIE1, sTIE2), soluble fms-like tyrosine kinase (sFlt-1), slit glycoprotein (Slt-2)/ Roundabout receptor (ROBO). The PCT levels will be batched and measured at the end of the study for the substudy assessing the association between PCT and clinical outcomes among patients receiving 7 vs 14 days of treatment. The results will not be made available to the treating team because this could unduly influence clinical practice and protocol adherence and is ethical because none of the participating sites are currently using PCT routinely. Following study completion, we will compare PCT area under the curve and day 7, 10 and 14 PCT levels among patients. We will also confirm whether 7 days of antibiotics is non-inferior to 14 days of antibiotics for bacteraemia, in subgroups with both normal and abnormal $(>0.25 \mu \mathrm{g} / \mathrm{mL})$ PCT levels on day 7 . We will conduct comparable analyses for the other inflammatory and angiogenic biomarkers.

\section{Microbiome substudy}

To assess the effect of shortening the duration of antibiotic administration on the human gut microbiome, we will collect rectal swabs from patients on the day of randomisation, and at days 7, 14 and 21 (or hospital discharge if earlier than day 21) from the start of appropriate antibiotic therapy. Flocked, sterile swabs will be inserted $2-3 \mathrm{~cm}$ past the anal verge, rotated three to four times, deposited into a room-temperature stable DNA/RNA preservative and stored at room temperature. No human sequence data will be obtained. Swabs will be transported for processing in batches, or at the end of the study, whichever is easier for the participating site. Samples will be processed for extraction of nucleic acid for $16 \mathrm{~S}$ rRNA microbiome sequencing and shotgun metagenomics sequencing. Participants who decline sample collection for the substudies will still be included in the main clinical trial.

\section{Protecting against sources of bias \\ Selection bias}

Selection bias (such as bias-by-indication or survival bias) will be minimised through rigorous concealed randomisation procedures. Although placebo controls have been used in some RCTs of antibiotic treatment duration, such as studies examining treatment duration for cellulitis, ${ }^{37}$ pyelonephritis ${ }^{38}$ and community-acquired pneumonia, ${ }^{40-43}$ they are not appropriate for bacteraemia treatment in acutely ill patients. It is not feasible to administer placebos for each of the many antimicrobials commonly used alone or in combination to treat the many aetiologies of bacteraemia. ${ }^{44}$ Even if it were possible to generate this many placebos, BALANCE patients are susceptible to developing secondary sources of nosocomial infection, and our preparatory work revealed that clinicians demand knowledge of whether a patient is receiving antibiotics or not.

\section{Outcome misclassification bias}

We have selected an objective primary outcome measure and we will use central adjudication committees blinded to treatment allocation for other outcomes including relapse, and secondary infection/colonisation with antibiotic-resistant organisms. ${ }^{45} 46$

\section{Withdrawal from study}

If a patient is withdrawn from the study prematurely, a withdrawal form will be completed. Data will be collected under the informed consent up to the point of a consent withdrawal. Among patients who withdraw consent for continuation of the trial, we will seek their consent to ascertain vital status at ICU and hospital discharge and at 90 days from the date of bacteraemia diagnosis. Anticipated reasons for withdrawal include patient not meeting inclusion criteria or having relevant exclusion criteria prior to randomisation, consent withdrawn by patient or substitute decision-maker, patient's physician believes patient should be withdrawn from the study and inadvertent duplicate randomisation. Detailed rationales for withdrawal will be recorded.

\section{Protocol adherence and protocol deviations}

We will define adherence to treatment duration protocol as receipt of $7 \pm 2$ days of adequate antibiotics in the shorter duration arm, and $14 \pm 2$ days in the longer duration arm; antibiotics stopped before or continued beyond these durations will be considered protocol deviations. We do not expect or aspire to achieve a $0 \%$ protocol deviation rate in this trial, given that some patients will develop persistent, recurrent or secondary infections for which antibiotics will need to be reinitiated or continued. It is appropriate for these patients to receive treatment as would occur outside of a trial. For example, in the multicentre PneumoA study of 8 vs 15 days of treatment for ventilator-associated pneumonia, protocol nonadherence was $18 \%$ in the 8-day treatment arm. ${ }^{17}$ Nonadherence rates have been as high as $50 \%$ seen in some studies of PCT-guided treatment for infections in critically ill patients. ${ }^{47}$ However, we will monitor protocol deviation rates overall and by hospital site during the trial, record rationales for the deviations, and strive to minimise unnecessary deviations.

\section{Frequency and duration of follow-up}

Patients will be reviewed daily in hospital for the first 14 days postrandomisation, and again at hospital discharge with extensive data collection (see case report form at balance.ccctg.ca). The research coordinator will contact the patient (or substitute decision-maker as appropriate) on day 90 by telephone to determine their disposition and vital status. Other sources of evidence for 90-day vital status will include clinical documentation of healthcare visits/readmissions and testing after 90 days.

\section{Primary outcome measure}

The primary outcome will be survival at 90 days from the date of bacteraemia, defined by the date of collection of 
the index positive blood culture. Although, most deaths from critical illness occur during hospital stay, lingering sequelae lead to a persistently elevated risk of death postdischarge. Therefore, we selected post-hospital 90-day mortality as a common vital status endpoint. ${ }^{48} 49$

\section{Secondary outcome measures}

The secondary outcomes include: (1) hospital mortality, (2) ICU mortality, (3) relapse rates of bacteraemia with the same organism, (4) antibiotic allergy and adverse events, (5) rates of C. difficile infection in hospital, (6) rates of secondary nosocomial infection/colonisation with antimicrobial-resistant organisms in hospital, (7) ICU length of stay, (8) hospital length of stay, (9) mechanical ventilation duration and (10) antibiotic-free days.

Antimicrobial-resistant organisms will be defined based on a positive routine culture yielding a highly resistant microbial organism as defined by the Dutch nosocomial infection surveillance guidelines. ${ }^{50}$ This broad definition includes methicillin-resistant $S$. aureus, vancomycinresistant enterococci, extended-spectrum beta-lactamase producing Enterobacteriaceae, carbapenem-resistant Gram-negative bacilli and multidrug-resistant Gramnegative bacilli (with definition of multidrug resistance differing according to Enterobacteriaceae and nonEnterobacteriaceae species).$^{50}$ We will also conduct a sensitivity analysis limited to isolation of these organism(s) only from sterile site specimens (such as blood, cerebrospinal fluid, peritoneal fluid, synovial fluid, pleural fluid and tissue biopsies).

Antibiotic-free days will be calculated as the number of days alive and not on any antibiotics in the time period from collection of the index blood culture to 28 days after this date; patients who die prior to day 28 will be assigned 0 antibiotic-free days.

\section{Statistical analysis}

\section{Sample size}

The primary analysis will assess whether 7 days of treatment is associated with non-inferior 90-day survival in comparison to 14 days. We require 1686 patients per arm to establish a non-inferiority margin of $-4 \%$ absolute decrement in survival ( (baseline mortality $22 \%)^{51}$ power $80 \%$, alpha 0.025 , one-sided equivalence test). We have inflated this to account for a maximum of $5 \%$ loss to follow-up and have incorporated early stopping rules to account for the three interim analyses (coefficient 1.017$)^{52} 53$ for a total sample size of 3626. Recent landmark trials in with similar baseline mortality rates have used $-4 \%$ as a non-inferiority $\operatorname{margin}^{54} 55$; the US Food and Drug Administration has recommended a similar margin for analogous industrysponsored trials. ${ }^{56}$ The PneumA study of 8-vs 15-day treatment for ventilator associated pneumonia (VAP) used a non-inferiority margin of $10 \%,{ }^{17}$ as have other recent prominent infectious diseases non-inferiority trials, ${ }^{20} 57$ but we believe lower non-inferiority margins are desirable, when feasible, for the outcome of survival. ${ }^{56}$

\section{Loss to follow-up}

We anticipate negligible loss of patients to follow-up. The Canadian Critical Care Trials Group (CCCTG) (www. ccctg.ca) has achieved virtually $100 \%$ follow-up to hospital discharge over all of its landmark RCTs ${ }^{58-60}$ Although we will be following survivors to ascertain 90-day mortality and relapse rates, we also expect close to $100 \%$ follow-up based on previous CCCTG experience and our own pilot RCT experience. ${ }^{32}{ }^{33}$ Nevertheless, we are accounting for up to $5 \%$ loss to follow-up in our sample size calculation.

\section{Analysis of primary outcome}

The BALANCE trial will be conducted, analysed and reported according to CONSORT (Consolidated Standards of Reporting Trials) guidelines, including analysing patients in the groups to which they were assigned (intention to treat) ${ }^{61}$ We will also include a per-protocol analysis. Inferences that 7-day treatment is non-inferior to 14-day treatment will be stronger if this finding is confirmed in both intention-to-treat and per-protocol analyses. ${ }^{62} \mathrm{We}$ will also perform a modified intention-to-treat (mITT) analysis, excluding patients who die before day 7 of treatment, given that these patients die prior to divergence in treatment assignment. ${ }^{63}$ The primary analysis will examine whether 90-day survival is non-inferior in the 7vs 14-day treatment group, as determined by whether the 95.7\% CI excludes a $4 \%$ absolute decrement in survival.

\section{Analysis of secondary outcomes}

Mortality rates at other time points will be calculated in a similar manner to 90-day mortality. We hypothesise that mortality rates will be non-inferior with 7 days of treatment. Continuous secondary outcomes, including lengths of stay in ICU and hospital, durations of ventilation and vasopressor use, and antibiotic-free days will be compared by the Wilcoxon test.

\section{Subgroup analyses}

The main subgroup analysis will be based on the underlying infectious syndrome causing bacteraemia (vascular catheter related, pneumonia, pyelonephritis, intraabdominal, skin and soft tissue, other identified source or unknown source). We will also perform subgroup analyses based on ICU versus non-ICU enrolments, community versus hospital acquisition, Gram-positive vs Gram-negative infection, illness severity (Acute Physiology And Chronic Health Evaluation (APACHE II) score of $\geq 25 \mathrm{vs}<25$ ) and vasopressor use on day of randomisation. We hypothesise that the non-inferiority of 7 vs 14 days of treatment will be consistent across these subgroups.

\section{Frequency of analyses}

Three interim analyses are planned for BALANCE at approximately 1/6 (600 patients), 1/3 (1200 patients) and 2/3 (2400 patients) of projected total enrolment; we will stop at the interim analysis for futility, inferiority or superiority using the O'Brien-Fleming spending function to generate adjusted CIs for the primary endpoint, splitting the type I error at $0.0000007,0.000452,0.013$ 
Interim Analysis 1: $\mathrm{N}$ per group=300

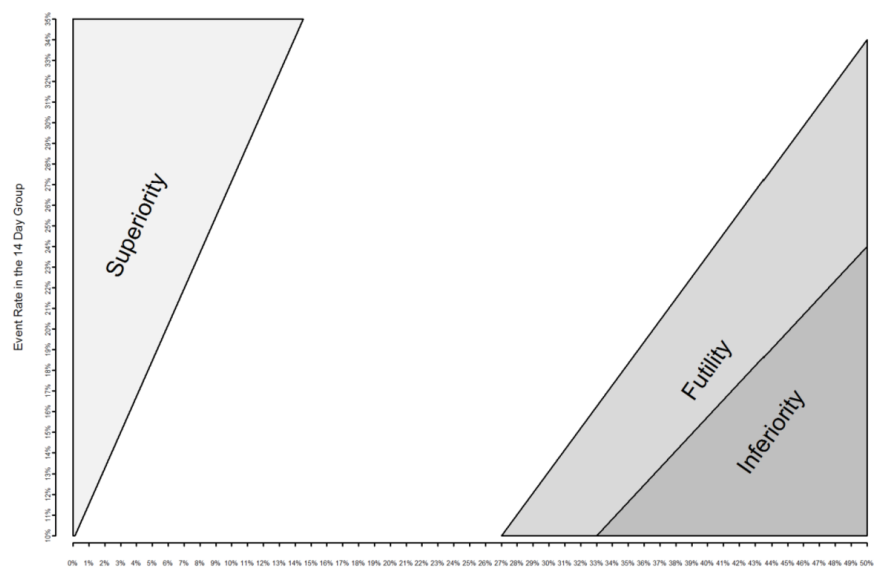

Event Rate in the 7 Day Group

Figure 2 Operationalising stopping guidelines for inferiority (dark grey shaded area), superiority (light grey shaded area) and futility (medium grey shaded area) with interim event rates at the first interim analysis $(n=600)$. Similar figures are available to the data monitoring committee for subsequent interim analyses.

and 0.043 with $99.99 \%, 99.95 \%, 98.68 \%$ and $95.70 \%$ twosided CIs to give an overall type I error of $2.5 \% .^{525362}$ The data monitoring committee (DMC) will be guided by a graphical plot indicating mortality differences which would meet futility, inferiority or superiority thresholds (figure 2). We will perform both frequentist-based and Bayesian-based analyses for endpoints at the study's termination. Subgroup analyses will not be performed for the interim analyses.

\section{Secondary Bayesian analysis}

Usual frequentist-based statistical analysis calculates the probability of obtaining data as extreme or more extreme than the observed data assuming the null hypothesis is true. Interpretations of clinical trials based on frequentist statistics using p-values and 95\% CIs can be challenging for clinicians for several reasons. First, frequentist-based analyses usually consider each analysis in isolation, without an easy mechanism for quantitatively incorporating prior information and without a true measure of the probability of clinical benefit. Quantitative interpretation of new information from clinical trials can be especially challenging when either prior evidence or perception does not align with new evidence. ${ }^{64-66}$ The interpretation of results of trials using a non-inferiority perspective can be additionally challenging, requiring interpretation of findings that may indicate non-inferiority, inferiority, superiority, equivalence or an inconclusive estimate of effect. ${ }^{67}$ Bayesian methods provide an alternative to null hypothesis statistical testing that allow quantification of evidence in favour of the null hypothesis, sequential testing and comparison of strength of evidence across different studies. ${ }^{68-71}$ In addition to our primary frequentist-based analysis of the primary 90-day mortality outcome, and secondary in-hospital and in-ICU mortality outcomes, we will additionally perform companion Bayesian analyses of each. This will be particularly informative should the study be either stopped for futility (a high likelihood of being unable to determine superiority, inferiority or noninferiority at planned or feasible samples sizes) to directly estimate the probability of treatment benefits. We will combine the data from BALANCE with a non-informative prior to deriving the posterior distribution based on which we will report the $95 \%$ credible intervals together with the probabilities of the difference in mortality between the two groups falling into the superiority, non-inferiority and inferiority regions.

\section{Steering committee}

The BALANCE steering committee is responsible for the development and oversight of the BALANCE RCT procedures, rigorous and ethical trial conduct, funding applications, advising the principal applicants on responses to questions from ethics boards, the DMC or other stakeholders, and eventual interpretation and compilation of study results into reports, scholarly manuscripts and knowledge translation (KT) and exchange activities. With BALANCE expansion to additional countries, additional steering committee member(s) will be added from each country with two or more enrolling sites and/ or has obtained regional grant funding to support the trial.

\section{Data monitoring ommittee}

The BALANCE DMC has multidisciplinary expertise in infectious diseases, critical care, RCT design and conduct, clinical epidemiology, biostatistics, interim analyses and early stopping rules. The BALANCE DMC charter (online supplementary file) is based on the Data Monitoring Committees: Lessons, Ethics, Statistics (DAMOCLES) Study Group charter, and draws heavily from the charter and experience of prior landmark CCCTG studies. ${ }^{58} 72$ At each interim analysis, the BALANCE principal investigators will provide the DMC with information on group characteristics, recruitment rates, adherence to treatment duration protocols, data completeness and accuracy, serious adverse events (SAEs), outcome event rates and co-enrolment prevalence. Data will be presented in both one-group or two-group tables in a manner that will prevent unmasking of group allocation to the research team. The DMC will be able to request an independent (not involved in the BALANCE trial) methods centre statistician to provide the unmasked group allocation, according to the BALANCE charter (online supplementary file), should that be deemed necessary by the DMC to interpret the interim analysis.

\section{Patient and public involvement}

The CCCTG includes a Patient and Family Partnership Committee (https://cccrpf.ca/) that has been engaged throughout BALANCE development and conduct. 


\section{ETHICS AND DISSEMINATION}

\section{Ethics approval}

Ethics approval has been obtained from the research ethics board of each participating site, along with central mechanisms in the Canadian provinces of Ontario and Quebec, the Australian states of New South Wales and Victoria and New Zealand (online supplementary file 2).

\section{Consent}

The research coordinator/site primary investigator will approach eligible patients (or their substitute decisionmakers) as soon as their blood cultures are positive to obtain informed consent. Enrolment can be delayed at maximum to the day 7 of adequate antibiotic treatment. Critically ill patients are frequently unable to provide initial consent due to altered level of consciousness or comprehension, and thus the CCCTG has standard operating procedures to seek assistance from substitute decisionmakers on behalf of patients. This process has been found feasible and acceptable to patients, decision-makers and research ethics boards across Canada. ${ }^{73-76}$ We will use this enhanced approach to consent, employing 13 previously described strategies distributed over three phases: preparation for the consent encounter, the consent encounter and follow-up to the consent encounter. ${ }^{77}$

\section{Expected adverse events}

Short-course (7 days) treatment duration could theoretically increase the risk of clinical treatment failure or relapse of the bloodstream infection or underlying focus of infection. Long-course (14 days) treatment on the other hand may increase the chance of resistance to antibiotics; occurrence of new antibiotic-resistant infections; $C$. difficile infection; and adverse events like allergy, anaphylaxis, antibiotic-related kidney injury, antibiotic-related hepatitis and other antibiotic-related organ toxicity. Our systematic review suggests that clinical cure and survival are similar among patients with bacteraemia receiving shorter and longer treatment, but these represent underpowered, post hoc subgroup analyses pooled from small trials. ${ }^{33}$ Any observational study assessing the impact of duration of treatment on patient outcomes would be limited by survivor bias (patients must survive long enough to be classified as receiving longer treatment) and indication bias (clinicians select sicker patients to receive longer duration treatment). Hence, patients and clinicians require a sufficiently powered RCT dedicated to answering the question of whether shorter treatments are effective for patients with bloodstream infection.

Morbidity and mortality are expected among patients with bloodstream infections. Accordingly, mortality at 90 days, in ICU and in hospital are trial outcomes, as are episodes of $C$. difficile colitis, and antibiotic-related allergy and adverse effects. Outcomes will be reported as such, rather than as SAEs, serious unexpected adverse reactions or suspected unexpected serious adverse reactions. These outcomes will be reported to the DMC at all interim analyses. We will closely monitor patient safety in the trial by recording the antimicrobial-related adverse events and serious unexpected adverse drug reactions, additionally interpreted by the steering committee and reported to the $\mathrm{DMC}$ at each interim analysis.

\section{Knowledge dissemination}

A major mandate of the CCCTG is translating knowledge into practice and advancing the science of KT in critically ill hospitalised patients. The study has also been endorsed by the Association of Medical Microbiology and Infectious Diseases Canada Clinical Research Network (AMMI Canada CRN), the Australian and New Zealand Intensive Care Society Clinical Trials Group (ANZICS-CTG) and the Australasian Society for Infectious Diseases Clinical Research Network (ASID-CRN). The involvement of the knowledge users and leaders in these organisations will lead to rapid national and international knowledge dissemination.

\section{DISCUSSION}

\section{Rationale for why the BALANCE trial is urgently needed}

The WHO, US Centers for Disease Control and Prevention, AMMI Canada and Health Canada have all declared antimicrobial resistance a global threat to health, ${ }^{1-4}$ based on rapidly increasing resistance rates and declining new drug development. ${ }^{5-7}$ The highest rates of antimicrobial resistance occur in hospitals, and ICUs in particular, and it is crucial that we develop data-informed mechanisms to decrease antimicrobial use and selection pressure. The vulnerability of acutely ill patients, the complexity of their treatments and the frequent uncertainty of their infectious syndromic diagnoses are all barriers to reducing antibiotic exposure in the ICU. It is very difficult to avoid initial broad-spectrum antibiotic treatment when acutely ill patients present with or develop definite or suspected infection. Multiple studies have demonstrated that early administration of effective antibiotics in the initial empiric window of antibiotic treatment is the strongest predictor of a favourable outcome in these patients. If empiric selections do not match the susceptibility profile of the isolated pathogen, the patient may be nearly twice as likely to die. ${ }^{78}$ Given that prevailing resistance rates are already high, broad-spectrum initial treatments are appropriate for many acutely ill patients. In contrast, it is much more feasible to reduce antibiotic use at the end of treatment courses, given that most patients may be treated longer than necessary, and excessive antibiotic durations are a top contributor to inappropriate antibiotic in all healthcare sectors. ${ }^{711-13}$ Shorter duration treatments have been demonstrated to be non-inferior to longer duration treatments for a range of infections. ${ }^{11} 19$ If BALANCE confirms this finding among patients with bacteraemia, it could result in effective but shorter prescribing practices for these patients. Shortening treatment durations should also reduce other adverse events, including C. difficile infections, and generate an estimated annual direct 
antimicrobial cost savings of \$C678-\$C798 million across North America and C\$1.4-1.6 billion across Europe. ${ }^{80}$

\section{Rationale for studying fixed duration therapy rather than individualised durations of treatment}

Ideally antibiotic treatment duration should be individualised, and each patient should receive exactly as much antibiotic treatment as needed until their infection is cured, and not longer. ${ }^{24} 81$ However, an RCT based on a clinical stopping rule may not be feasible in acutely ill patients, since there are currently no proven accurate measures of cure versus persistent infection. The challenge in diagnosing and monitoring infection in ICU has sparked studies of novel biomarkers to guide antibiotic treatment duration. ${ }^{43}$ One biomarker, PCT, has been used successfully to reduce average treatment durations in sepsis. ${ }^{43}$ However, follow-up meta-analyses have indicated that the bacteraemic subgroups in PCT trials have tended to receive prolonged treatment durations, ${ }^{82}$ perhaps because of high non-adherence rates to algorithm-guided treatment. ${ }^{47}$ Therefore, we have designed a randomised trial of fixed shorter versus longer duration antibiotic therapy, guided by our preparatory studies, as the most easily transferable result to immediately inform clinical practice. This approach has been successful in more than two dozen randomised controlled trials of infectious diseases that are potentially complicated by bacteraemia. ${ }^{19}$ However, we appreciate the future promise of biomarkers to add nuance to individualised treatment decisions, and so in a nested substudy, we will measure PCT and other novel biomarker levels and trajectory in both treatment arms to see if they could provide incremental value. ${ }^{61}$

\section{TRIAL STATUS}

The BALANCE pilot RCT was launched in October 2014 at the central study site, Sunnybrook Health Sciences Centre, expanded to include a total of 10 CCCTG sites across Canada and served as a successful vanguard for this BALANCE main RCT. An additional 26 Canadian sites have joined BALANCE, for a total of 36 Canadian sites in six provinces. A parallel BALANCE pilot RCT on medical and surgical wards was launched in October 2016 at a subset of six BALANCE sites, which confirmed the feasibility of recruitment and protocol adherence on non-ICU wards ${ }^{33}$ and enabled hospital-wide expansion; approximately half of sites have now opted for hospitalwide enrolments. We have expanded BALANCE internationally to include sites in Australia (6), New Zealand (10), Saudi Arabia (2), Israel (1) and the USA (2). Therefore, there are currently 57 sites enrolling patients into BALANCE, and with continued expansion we anticipate approximately 70 sites prior to study completion (anticipated for 2022-2023). The BALANCE DMC conducted the first interim analysis $(\mathrm{n}=600)$ on 30 September 2019 and recommended continuing enrolment. As of 13 February 2020, a total of 1447 patients have been recruited into BALANCE, and data from the first 1200 patients are being analysed for the DMC review of the second interim analysis.

\section{Author affiliations}

${ }^{1}$ Division of Infectious Diseases \& Clinical Epidemiology, Sunnybrook Health Sciences Centre, University of Toronto, Toronto, Ontario, Canada

${ }^{2}$ Institute for Clinical Evaluative Sciences, Sunnybrook Health Sciences Centre, Toronto, Ontario, Canada

${ }^{3}$ Department of Critical Care Medicine, Sunnybrook Health Sciences Centre, Toronto, Ontario, Canada

${ }^{4}$ Intensive Care Department, King Saud Bin Abdulaziz University for Health Sciences, Riyadh, Saudi Arabia

${ }^{5}$ McMaster University, Hamilton, Ontario, Canada

${ }^{6}$ Departments of Critical Care Medicine and Anesthesiology, Pain Management and Perioperative Medicine, Dalhousie University, Halifax, Nova Scotia, Canada

${ }^{7}$ Auckland City Hospital, Auckland, New Zealand

${ }^{8}$ Kingston General Hospital, Kingston, Ontario, Canada

${ }^{9}$ The University of Auckland, Auckland, New Zealand

${ }^{10}$ Royal Columbian Hospital, New Westminster, British Columbia, Canada

${ }^{11}$ Centre for Inflammatory Diseases, Monash University School of Clinical Sciences, Melborne, Victoria, Australia

${ }^{12}$ Critical Care and Perioperative Medicine, School of Clinical Sciences, Monash University and Monash Health, Melbourne, Victoria, Australia

${ }^{13}$ Departments of Medicine and Critical Care Medicine, Sunnybrook Health Sciences Centre, Toronto, Ontario, Canada

Acknowledgements The authors thank the CCCTG for facilitating the entire BALANCE research programme and the membership for providing constructive feedback at thrice yearly meetings, as well as manuscript and grant review. We also thank the AMMI Canada CRN, ANZICS-CTG and ASID-CRN for endorsing BALANCE and infectious diseases and intensive care community engagement in all participating regions. We would like to acknowledge the site investigators in Canada (John Marshall, Michael Detsky, Elizabeth Wilcox, Bryan Coburn, Phil Shin, Robert Cirone, Janos Pataki, Nava Maham, Alexandra Binnie, Emilie BelleyCote, Richard Whitlock, Jennifer Tsang, Erick Duan, Brenda Reeve, Cory Scholes, Claudio Martin, Lauralyn McIntyre, Navdeep Mehta, Francois Lamontagne, Francois Lauzier, Maude St-Onge, Pierre Aslanian, Emmanuel Charbonney, Han Ting Wang, François Lellouche, Kosar Khwaja, Salman Qureshi, Anand Kumar, Tom Stelfox, Sean Bagshaw, Donald Griesdale, Gordon Wood, Osama Loubani, Linda Taggert, Andrew Morris, Pavani Das, Mark Downing, Chris Graham, Alicia Sarabia, Tom Havey, Kevin Woodward, Neal Irfan, Ali Firdous, Tom Szakacs, Sameer El Sayed, Gerald Evans, Derek Macfadden, Roger Sandre, Alex Carignan, Julie BestmanSmith, Valérie Martel-Laferrière, Andre Poirier, Christian Lavallee, Todd Lee, John Conly, Wendy Sligl, Jennifer Grant and Lynn Johnston); Australia (Gopal Taori, Vineet Sarode, David Brewster, Sam Rudham, Gururaj Nagaraj, Vineet Nayyar, Pierre Janin, James Winearls, Kylie Horne, Amalie Wilkie, Debbie Marriott, Keat Choong, Jonathan Iredell, Bernard Hudson, John Gerrard and Paul Griffin); New Zealand (Colin McArthur, David Knight, Ross Freebairn, Jonathan Albrett, Paul Young, Alex Kazemi, Andrew Stapleton, Ulrike Buehner, Robert Martynoga, Sally Roberts, Sarah Metcalf, Andrew Burns, Maxim Bloomfield and Christopher Hopkins); Saudi Arabia (Basem Alraddadi); Israel (Dafna Yahav and llya Kagan) and the USA (Abhijit Duggal, Vikramjit Mukherjee, Laura Evans and David Kaufman). We are deeply grateful for the hard work of the individual research coordinators at each site, including: Orla Smith, Gyan Sandhhu, Jennifer Hodder, Marlene Santos, Sumesh Shah, Karolina Walczak, Maria Kulikova, Rizani Ravindran, Alexandra Lostun, Kanthi Kavikondala, Gloria Crowl, Mobina Khurram, Noha Aref, Zaynab Panchbhaya, France Clarke, Nevena Savija, Courtney Mullen, Mercedes Camargo, Will Dechert, Eileen Campbell, Athena Ovsenek, Miranda Hunt, llinca Georgescu, Irene Watpool, Rebecca Porteous, Brigette Gomes, Shelley Acres, Kaitlyn Montroy, Louis Lakatos, Joannie Marchand, Élaine Carbonneau, David Bellemare, Gabrielle Guilbault, Estel Duquet, Ali Ghamraoui, Martine Lebrasseur, Danielle Tapps, Danae Tassy, Patricia Lizotte, Josie Campisi, Norine Alam, Nicole Marten, Justin Lys, Stacy Ruddell, Stacy Ruddell, Nadia Baig, Lorena McCoshen, Suzette Willems, Denise Foster, Gayle Carney, Laura Magennis, Omar Mehkri, Andrei Hastings, Ashley Witzl, Eman Al Qasim, Rawan Alsaadi, Lama Hefni, Adi Turjeman, Eileen Gilder, Magdalena Butler, Keri-Anne Cowdrey, Samantha Ryan, Philippa Neal, Lynette Newby, Rachael McConnochie, Yan Chen, Catherine Simmonds, Jan Mehrtens, Anna Morris, Kate Miller, Emmeline Minto, Kim Parker, Stacey Morgan, Carolyn Jackson, Raulle Cruz, Cassie Lawrence, Agnes McKay, Charlotte Latimer-Bell, Hannah Smellie, Harriet Judd, Samantha 
Edney, Nina Beehre, Yvonne Robertson, Anna Hunt, Georgia Hill, Rima Song, Dinu Girijadevi, Erin Williams, Kara Trask, Sarah Rogers, Llesley Chadwick, Penelope Park, Christine Rolls, Liz Thomas, Carmel Chapman, Dhiraj Dwivedi, Chloe Peppin, Fareda Fazli, Katherine Shepherd, Nicole Percy, Shannon Simpson, Claire Reynolds, Lauren Murray, Lorretta Forbes, Jane Brailsford, Teena Maguire, Jing Kong, Elizabeth Yarad, Naomi Hammond, Frances Bass, Mandy Tallot, Megan Martin, Julie Smith, Madeline Eyles, Anna Smith, Gabrielle Hanlon, Roberta Littleford, Kellie Schneider and Lynette Morrison).

Contributors ND and RAF conceived the research question. ND and RAF designed the study, with crucial inputs from AHR, RP, YMA, DJC, RH, SPM, JM, RP, SR, BR and YS. ND and RAF drafted the manuscript with important revisions provided by AHR, RP, YMA, DJC, RH, SPM, JM, RP, SR, BR and YS.

Funding The BALANCE RCT is supported by a Project Grant from the Canadian Institutes of Health Research (CIHR), as well as grants from the New Zealand Health Research Council, and the Australian National Medical Health Research Council. BALANCE preparatory work and substudies have also received support from CIHR as well as Physicians Services Incorporated, the Canadian Frailty Network and The Ontario Ministry of Health and Long Term Care (MOHLTC) Alternate Funding Plan Innovation Fund Award.

\section{Competing interests None declared.}

Patient and public involvement Patients and/or the public were involved in the design, conduct, reporting or dissemination plans of this research. Refer to the Methods and analysis section for further details.

\section{Patient consent for publication Not required.}

Provenance and peer review Not commissioned; peer reviewed for ethical and funding approval prior to submission.

Open access This is an open access article distributed in accordance with the Creative Commons Attribution Non Commercial (CC BY-NC 4.0) license, which permits others to distribute, remix, adapt, build upon this work non-commercially, and license their derivative works on different terms, provided the original work is properly cited, appropriate credit is given, any changes made indicated, and the use is non-commercial. See: http://creativecommons.org/licenses/by-nc/4.0/.

\section{ORCID iD}

Nick Daneman http://orcid.org/0000-0001-8827-3764

\section{REFERENCES}

1 Goto M, Al-Hasan MN. Overall burden of bloodstream infection and nosocomial bloodstream infection in North America and Europe. Clin Microbiol Infect 2013;19:501-9.

2 Vincent J-L, Rello J, Marshall J, et al. International study of the prevalence and outcomes of infection in intensive care units. JAMA 2009;302:2323-9.

3 Renaud B, Brun-Buisson C. Outcomes of primary and catheterrelated bacteremia. A cohort and case-control study in critically ill patients. Am J Respir Crit Care Med 2001;163:1584-90.

4 Pittet D, Tarara D, Wenzel RP. Nosocomial bloodstream infection in critically ill patients. excess length of stay, extra costs, and attributable mortality. JAMA 1994;271:1598-601.

5 Laupland KB, Lee H, Gregson DB, et al. Cost of intensive care unitacquired bloodstream infections. J Hosp Infect 2006;63:124-32.

6 Yamashita SK, Louie M, Simor AE, et al. Microbiological surveillance and parenteral antibiotic use in a critical care unit. Can J Infect Dis 2000;11:107-11.

7 Hecker MT, Aron DC, Patel NP, et al. Unnecessary use of antimicrobials in hospitalized patients: current patterns of misuse with an emphasis on the antianaerobic spectrum of activity. Arch Intern Med 2003;163:972-8.

8 Dellit TH, Owens RC, McGowan JE, et al. Infectious diseases Society of America and the Society for healthcare epidemiology of America guidelines for developing an institutional program to enhance antimicrobial stewardship. Clin Infect Dis 2007;44:159-77.

9 Shehab N, Patel PR, Srinivasan A, et al. Emergency department visits for antibiotic-associated adverse events. Clin Infect Dis 2008;47:735-43.

10 Gholami K, Parsa S, Shalviri G, et al. Anti-infectives-induced adverse drug reactions in hospitalized patients. Pharmacoepidemiol Drug Saf 2005;14:501-6.

11 Rice LB. The Maxwell Finland Lecture: for the duration-rational antibiotic administration in an era of antimicrobial resistance and Clostridium difficile. Clin Infect Dis 2008;46:491-6.
12 Hurley HJ, Knepper BC, Price CS, et al. Avoidable antibiotic exposure for uncomplicated skin and soft tissue infections in the ambulatory care setting. Am J Med 2013;126:1099-106.

13 Daneman N, Gruneir A, Bronskill SE, et al. Prolonged antibiotic treatment in long-term care: role of the prescriber. JAMA Intern Med 2013;173:673-82.

14 Stevens V, Dumyati G, Fine LS, et al. Cumulative antibiotic exposures over time and the risk of Clostridium difficile infection. Clin Infect Dis 2011;53:42-8.

15 Johnson MT, Reichley R, Hoppe-Bauer J, et al. Impact of previous antibiotic therapy on outcome of gram-negative severe sepsis. Crit Care Med 2011;39:1859-65.

16 Rafailidis PI, Pitsounis Al, Falagas ME. Meta-Analyses on the optimization of the duration of antimicrobial treatment for various infections. Infect Dis Clin North Am 2009;23:269-76.

17 Chastre J, Wolff M, Fagon J-Y, et al. Comparison of 8 vs 15 days of antibiotic therapy for ventilator-associated pneumonia in adults: a randomized trial. JAMA 2003;290:2588-98.

18 Martin CM, Priestap F, Fisher $\mathrm{H}$, et al. A prospective, observational registry of patients with severe sepsis: the Canadian sepsis treatment and response registry. Crit Care Med 2009;37:81-8.

19 Havey TC, Fowler RA, Daneman N. Duration of antibiotic therapy for bacteremia: a systematic review and meta-analysis. Crit Care 2011;15:R267.

20 Yahav D, Franceschini E, Koppel F, et al. Seven versus fourteen days of antibiotic therapy for uncomplicated gram-negative bacteremia: a non-inferiority randomized controlled trial. Clin Infect Dis 2018.

21 Daneman N, Fowler RA. Shortening antibiotic treatment durations for bacteremia. Clin Infect Dis 2019;69:1099-100.

22 Mandell LA, Wunderink RG, Anzueto A, et al. Infectious diseases Society of America/American thoracic Society consensus guidelines on the management of community-acquired pneumonia in adults. Clin Infect Dis 2007;44:S27-72.

23 American Thoracic Society, Infectious Diseases Society of America. Guidelines for the management of adults with hospital-acquired, ventilator-associated, and healthcare-associated pneumonia. Am J Respir Crit Care Med 2005;171:388-416.

24 Solomkin JS, Mazuski JE, Bradley JS, et al. Diagnosis and management of complicated intra-abdominal infection in adults and children: guidelines by the surgical infection Society and the infectious diseases Society of America. Clin Infect Dis 2010;50:133-64.

25 Mermel LA, Allon M, Bouza E, et al. Clinical practice guidelines for the diagnosis and management of intravascular catheter-related infection: 2009 update by the infectious diseases Society of America. Clin Infect Dis 2009;49:1-45.

26 Gupta K, Hooton TM, Naber KG, et al. International clinical practice guidelines for the treatment of acute uncomplicated cystitis and pyelonephritis in women: a 2010 update by the infectious diseases Society of America and the European Society for microbiology and infectious diseases. Clin Infect Dis 2011;52:e103-20.

27 Stevens DL, Bisno AL, Chambers HF, et al. Practice guidelines for the diagnosis and management of skin and soft-tissue infections. Clin Infect Dis 2005;41:1373-406.

28 Daneman N, Shore K, Pinto R, et al. Antibiotic treatment duration for bloodstream infections in critically ill patients: a national survey of Canadian infectious diseases and critical care specialists. Int $J$ Antimicrob Agents 2011;38:480-5.

29 Alwan M, Davis JS, Daneman N, et al. Duration of therapy recommended for bacteraemic illness varies widely amongst clinicians. Int J Antimicrob Agents 2019;54:184-8.

30 Havey T, Fowler R, Pinto R, et al. Duration of antibiotic therapy in intensive care unit bloodstream infections. Can J Infect Dis Med Microbiol 2013;24:129-37.

31 Daneman N, Rishu AH, Xiong W, et al. Antibiotic treatment durations among Canadian critically ill patients with bacteremia. In Submission AJRCCM 2014

32 Daneman N, Rishu AH, Pinto R, et al. 7 versus 14 days of antibiotic treatment for critically ill patients with bloodstream infection: a pilot randomized clinical trial. Trials 2018;19:111.

33 Daneman N, Rishu AH, Pinto R, et al. A pilot randomized controlled trial of 7 versus 14 days of antibiotic treatment for bloodstream infection on non-intensive care versus intensive care wards. Trials 2020;21:92

34 Clinical and Laboratory Standards Institute. Principles and procedures for blood Cutlures; Approved guideline M47-A. No. 17. 201127.

35 Thwaites GE, Edgeworth JD, Gkrania-Klotsas E, et al. Clinical management of Staphylococcus aureus bacteraemia. Lancet Infect Dis 2011;11:208-22. 
36 Daneman N, Rishu AH, Xiong W, et al. Bacteremia Antibiotic Length Actually Needed for Clinical Effectiveness (BALANCE): study protocol for a pilot randomized controlled trial. Trials 2015;16:173.

37 Hepburn MJ, Dooley DP, Skidmore PJ, et al. Comparison of shortcourse ( 5 days) and standard ( 10 days) treatment for uncomplicated cellulitis. Arch Intern Med 2004;164:1669-74.

38 Sandberg T, Skoog G, Hermansson AB, et al. Ciprofloxacin for 7 days versus 14 days in women with acute pyelonephritis: a randomised, open-label and double-blind, placebo-controlled, noninferiority trial. Lancet 2012;380:484-90.

39 Jernelius $\mathrm{H}$, Zbornik J, Bauer CA. One or three weeks' treatment of acute pyelonephritis? A double-blind comparison, using a fixed combination of pivampicillin plus pivmecillinam. Acta Med Scand 1988;223:469-77.

40 Agarwal G, Awasthi S, Kabra SK, et al. Three day versus five day treatment with amoxicillin for non-severe pneumonia in young children: a multicentre randomised controlled trial. BMJ 2004;328:791.

41 Pakistan Multicentre Amoxycillin Short Course Therapy (MASCOT) pneumonia study group. Clinical efficacy of 3 days versus 5 days of oral amoxicillin for treatment of childhood pneumonia: a multicentre double-blind trial. Lancet 2002;360:835-41.

42 Dunbar LM, Wunderink RG, Habib MP, et al. High-Dose, short-course levofloxacin for community-acquired pneumonia: a new treatment paradigm. Clin Infect Dis 2003;37:752-60.

43 Tellier G, Niederman MS, Nusrat R, et al. Clinical and bacteriological efficacy and safety of 5 and 7 day regimens of telithromycin once daily compared with a 10 day regimen of clarithromycin twice daily in patients with mild to moderate community-acquired pneumonia. $J$ Antimicrob Chemother 2004;54:515-23.

44 Craig J, Daneman N, Rishu A, et al. Measuring the duration of adequate antimicrobial treatment in critical care. $J$ Thorac Dis 2014;189:A3120.

45 Arnold DM, Lauzier F, Rabbat C, et al. Adjudication of bleeding outcomes in an international thromboprophylaxis trial in critical illness. Thromb Res 2013;131:204-9.

46 Cook D, Walter S, Freitag A, et al. Adjudicating ventilator-associated pneumonia in a randomized trial of critically ill patients. $J$ Crit Care 1998;13:159-63.

47 Bouadma L, Luyt C-E, Tubach F, et al. Use of procalcitonin to reduce patients' exposure to antibiotics in intensive care units (PRORATA trial): a multicentre randomised controlled trial. Lancet 2010;375:463-74.

48 Hua M, Wunsch $\mathrm{H}$. Reporting data on long-term follow-up of critical care trials. Thorax 2016;71:395-6.

49 Blackwood B, Ringrow S, Clarke M, et al. A core outcome set for critical care ventilation trials. Crit Care Med 2019;47:1324-31.

50 de Smet AMGA, Kluytmans JAJW, Blok HEM, et al. Selective digestive tract decontamination and selective oropharyngeal decontamination and antibiotic resistance in patients in intensivecare units: an open-label, clustered group-randomised, crossover study. Lancet Infect Dis 2011;11:372-80.

51 Daneman N, Rishu AH, Xiong W, et al. Duration of antimicrobia treatment for bacteremia in Canadian critically ill patients. Crit Care Med 2016;44:256-64.

52 O'Brien PC, Fleming TR. A multiple testing procedure for clinical trials. Biometrics 1979;35:549-56.

53 Jennison C, Turnbull B. Group sequential methods with applications to clinical trials. Chapman and Hall, 2015.

54 Finfer S, Bellomo R, Boyce N, et al. A comparison of albumin and saline for fluid resuscitation in the intensive care unit. $N$ Engl $\mathrm{J} \mathrm{Med}$ 2004;350:2247-56

55 Hébert PC, Wells G, Blajchman MA, et al. A multicenter, randomized, controlled clinical trial of transfusion requirements in critical care. transfusion requirements in critical care Investigators, Canadian critical care Trials Group. N Engl J Med 1999;340:409-17.

56 United States Deaprtment of Health and Human Services. Guidance for industry non-inferiority clinical trials 2010

57 Iversen K, Ihlemann N, Gill SU, et al. Partial oral versus intravenous antibiotic treatment of endocarditis. N Engl J Med 2019;380:415-24.

58 PROTECT Investigators for the Canadian Critical Care Trials Group and the Australian and New Zealand Intensive Care Society
Clinical Trials Group, Cook D, Meade M, et al. Dalteparin versus unfractionated heparin in critically ill patients. N Engl J Med 2011;364:1305-14.

59 Ferguson ND, Cook DJ, Guyatt GH, et al. High-Frequency oscillation in early acute respiratory distress syndrome. $N$ Engl J Med 2013;368:795-805.

60 Canadian Critical Care Trials Group. A randomized trial of diagnostic techniques for ventilator-associated pneumonia. N Engl J Med 2006;355:2619-30.

61 Moher D, Hopewell S, Schulz KF, et al. Consort 2010 explanation and elaboration: updated guidelines for reporting parallel group randomised trials. BMJ 2010;340:c869.

62 Mulla SM, Scott IA, Jackevicius CA, et al. How to use a noninferiority trial: users' guides to the medical literature. JAMA 2012:308:2605-11.

63 Fergusson D, Aaron SD, Guyatt G, et al. Post-Randomisation exclusions: the intention to treat principle and excluding patients from analysis. BMJ 2002;325:652-4.

64 Goligher EC, Tomlinson G, Hajage D, et al. Extracorporeal membrane oxygenation for severe acute respiratory distress syndrome and posterior probability of mortality benefit in a post hoc Bayesian analysis of a randomized clinical trial. JAMA 2018;320:2251-9.

65 Spiegelhalter DJ, Abrams KR. Bayesian approaches to clinical trials and health-care evaluation. John Wiley \& Sons, 2004.

66 Aberegg SK. Post hoc Bayesian analyses. JAMA 2019;321:1631-2.

67 Piaggio G, Elbourne DR, Pocock SJ, et al. Reporting of noninferiority and equivalence randomized trials: extension of the CONSORT 2010 statement. JAMA 2012;308:2594-604.

68 Li W, Chen M-H, Wangy X, et al. Bayesian design of non-inferiority clinical trials via the Bayes factor. Stat Biosci 2018:10:439-59.

69 van Ravenzwaaij D, Monden R, Tendeiro JN, et al. Bayes factors for superiority, non-inferiority, and equivalence designs. BMC Med Res Methodol 2019;19:71.

70 Berry DA. Bayesian clinical trials. Nat Rev Drug Discov 2006;5:27-36.

71 Zohar S, Latouche A, Taconnet M, et al. Software to compute and conduct sequential Bayesian phase I or II dose-ranging clinical trials with stopping rules. Comput Methods Programs Biomed 2003;72:117-25

72 DAMOCLES Study Group, NHS Health Technology Assessment Programme. A proposed charter for clinical trial data monitoring committees: helping them to do their job well. Lancet 2005;365:711-22.

73 Duffett M, Burns KE, Kho ME, et al. Consent in critical care trials: a survey of Canadian research ethics boards and critical care researchers. J Crit Care 2011;26:533.e11-22.

74 Scales DC. Research to inform the consent-to-research process. Intensive Care Med 2013;39:1484-6.

75 Smith OM, McDonald E, Zytaruk N, et al. Enhancing the informed consent process for critical care research: strategies from a thromboprophylaxis trial. Intensive Crit Care Nurs 2013.

76 Smith OM, McDonald E, Zytaruk N, et al. Rates and determinants of informed consent: a case study of an international thromboprophylaxis trial. J Crit Care 2013;28:28-39.

77 Smith OM, McDonald E, Zytaruk N, et al. Enhancing the informed consent process for critical care research: strategies from a thromboprophylaxis trial. Intensive Crit Care Nurs 2013;29:300-9.

78 Paul M, Shani V, Muchtar E, et al. Systematic review and metaanalysis of the efficacy of appropriate empiric antibiotic therapy for sepsis. Antimicrob Agents Chemother 2010;54:4851-63.

79 Kumar A, Roberts D, Wood KE, et al. Duration of hypotension before initiation of effective antimicrobial therapy is the critical determinant of survival in human septic shock. Crit Care Med 2006;34:1589-96.

80 Daneman N, Rishu A, Xiong W, et al. Antimicrobial cost savings associated with shorter duration treatment for bloodstream infections. JAMMI 2017;1:32-4.

81 Llewelyn MJ, Fitzpatrick JM, Darwin E, et al. The antibiotic course has had its day. $B M J$ 2017;358:j3418.

82 Meier MA, Branche A, Neeser OL, et al. Procalcitonin-guided antibiotic treatment in patients with positive blood cultures: a patientlevel meta-analysis of randomized trials. Clin Infect Dis 2018. 